\title{
L'évolution du sacré chez Pontus de Tyard
}

\author{
EVA KUSHNER
}

Dans les grandes lignes, la vie de Tyard se scinde en deux périodes principales, de part et d'autre de son accession à l'évêché de Chalon. Le poètephilosophe fut avant tout le Solitaire de Bissy-sur-Fley, qui toutefois se laissa attirer plus fréquemment par Paris que par Lyon à partir de 1569 (la cour, le salon de Mme de Retz, l'Académie ... ). Toute l'œuvre poétique de Tyard existe avant qu'il ne soit évêque de Chalon, c'est-à-dire avant 1578, à l'exception des Douze fables defleuves et fontaines qui, composées en 1555, ne paraissent qu'en 1586: c'est dire que notre auteur renonce à la poésie après les Nouvell' ceuvres poétiques (1573). Le philosophe reprendra la plume au cours des dernières années de sa vie avec, notamment, le De recta nominum impositione de 1603; mais ce sont ses homélies qui forment l'œuvre majeure de l'évêque. Il s'agit de quatre suites d'homélies dont nous dirons ailleurs ${ }^{1}$ la remarquable ordonnance; ce furent d'abord les Homilies ou Discours sur l'oraison dominicale (Paris: Mamert Patisson, 1585); puis les Homilies, ou Contemplations sur la Passion de Nostre Sauveur, Jesus-Christ (Paris: Mamert Patisson, 1586). La même année parurent les Trois livres d'homilies contenant, outre la réédition des deux ouvrages précédents, un Troisième livre des Homilies de M. Pontus de Tyard sur la dignité de la Croix. Enfin, Tyard publie les Homilies sur la premiere table du decalogue (Paris, Claude Chappelet, 1588).

Il serait donc aisé de diviser l'œuvre de Tyard en une partie "profane" et une partie "sacrée". L'une, dans le sillage de la Pléiade paganisante, serait celle de la poésie amoureuse; l'autre incarnerait les convictions et les préoccupations religieuses, voire paroissiales de l'évêque de Chalon. La division des genres confirmerait celle qu'impose la chronologie: les genres lyriques, et en particulier le sonnet si prédominant dans les Erreurs amoureuses, ne chanteraient d'autre divinité que Vénus, nom si galvaudé que depuis longtemps aucune sacralité ne s'y attachait; les homélies auraient un statut fonctionnel: susciter le repentir et la foi chez l'auditeur. Tyard aurait ainsi sacrifié tout souci esthétique sur l'autel de la ContreRéforme....

Or, l'unité interne de l'œuvre tyardienne s'avère plus forte que la coupure de 1578: l'exigence religieuse préexiste longuement à la conversion de 
l'homme de lettres en homme d'église; elle ne cesse d'accompagner et d'imprégner le "cornucopian text", enrichissant et compliquant l'apport de l'imaginaire plutôt que de le contrecarrer; et, en dépit de la différence de leurs contenus, les textes des deux périodes sont liés par certaines insistances au niveau des symboles et des thèmes, fondements d'une unité sousjacente sous le signe du sacré. Déjà, le chantre de Pasithée dote la Beauté d'un sens véritablement religieux, à l'instar d'ailleurs de Maurice Scève, qui s'écrie dans Delie:

Me pourra donc estre imputé à vice

Constituant en elle mes haults Cieulx?

(LXXV)

ou encore:

Me ravissant ta divine harmonie

Souventesfois jusques aux Cieulx me tire ... ${ }^{3}$

(CLVII)

Dans le sillage d'un certain pétrarquisme de telles expressions constituent sans doute des équivalents métaphoriques de l'admiration, voire de la vénération que l'amant voue à sa dame; chez Tyard toutefois elles se relient à tout un réseau d'images révélant une vision de la beauté qui, par l'intermédiaire d'un être humain, ne saurait mener qu'à Dieu même:

Quand je m'eslieve en contemplation,
M'esmerveillant de ce divin visage,
Saint et divin, contre mortel usage,
Fait au pourtrait de la perfection,
Soudain l'ardeur de serve passion
M...
Fe prosternant aux piedz de cette image,
Fond mon desir tout en devotion.
Ce saint seul Dieu de ma félicité
Que mon coeur las mourant à son service
S'offre aux autelz de mon idolatrie. ${ }^{4}$

(I/V)

Pasithée est transformée en objet d'adoration religieuse, et c'est de cette transformation même qu'il est question dans ce poème. L'acte de contemplation "fond" le désir en dévotion: c'est dire que le désir n'est pas nié mais qu'il est entraîné dans et par la contemplation et transfiguré (sublimé, dirait-on aujourd'hui). Parallélisme, donc, de l'expérience mystique et de l'expérience amoureuse; ou faut-il aller plus loin et affirmer que l'expérience mystique a pris toute la place? Cela serait légitime car la dame 
elle-même est transfigurée en objet d'adoration et elle n'est plus que cela dans le poème. Elle est "divin visage, / Saint et divin" et son être est entièrement absorbé en et par ce mode d'existence hiératique; ce que souligne l'expression "contre mortel usage": la Dame est ici séparée tant de sa propre identité dans le monde réel que de son statut d'objet d'un simple amour humain. Ce qu'adore le sujet lyrique dans les tercets, "ce Saint seul Dieu de ma félicité", est caractéristiquement au masculin; certes, il s'agit du "divin visage" mentionné beaucoup plus tôt dans le poème; mais, à moins de remonter jusqu'à cet antécédent, le lecteur perçoit une divinité elle-même masculine. La transfiguration de l'objet aimé a résorbé sa féminité. Déjà, dans la poésie lyrique médiévale, la femme était souvent "le seigneur". Le sujet lyrique recrée l'objet de son amour à la mesure de son besoin d'adoration et, ce faisant, il lui substitue l'objet divin de toute adoration.

Affirmer que la poésie amoureuse de Tyard participe globalement à . cette expérience spirituelle serait inexact: il arrive fréquemment que les attributs divins de l'aimée, la nomenclature qui la désigne (par exemple "ma Deesse"), les symboles cosmiques s'attachant à elle, et les émotions suscitées chez son adorateur, ne relèvent que métaphoriquement du sacré, rejoignant ainsi tout un aspect bien connu du style pétrarquisant. Le sonnet I/V représente donc un cas-limite où la conscience du sacré occupe l'espace poétique entier. À la fin du Troisième livre des Erreurs amoureuses, qui est aussi la fin du cycle entier, le sonnet XXXIII incarne à première vue une situation semblable, car tout l'espace du poème y est voué à l'adoration. Pourtant, il s'avère que la relation entre l'adorateur et l'adorée a subi une profonde transformation, qui correspond au trajet poétique entre le premier et le troisième livre. Ici, en effet, le "je" lyrique, loin de s'absorber dans l'objet de son amour, oppose à celui-ci sa propre réalité, voire sa propre résistance. Il ne s'agit plus d'une adoration soumise à l'Autre mais d'une offrande, sinon d'égal à égal, du moins d'un être maintenant conscient de sa propre valeur, à un autre, infiniment parfait encore, mais moins distant qu'auparavant. Seconde différence: au bout de sa longue errance le Solitaire chante à sa divine adorée, qui pourtant lui restera interdite à jamais, un hymne de bonheur, ponctué, avec insistance, par l'adverbe "heureusement".

Mon esprit ha heureusement porté

Au plus beau Ciel sa force outrecuidée,

Pour s'abbreuver en la plus belle Idée

D'où le pourtrait j'ay pris de ta beauté.

Heureusement mon coeur s'est enretté

Dans ta beauté d'un libre oeil regardée:

Et ma foy t'est heureusement gardée,

Et t'a ma bouche heureusement chanté: 
Mais si encor heureusement t'espere Qu'en fin ton cours (ô ma divine Sphere)

Veut asseurer la crainte, qui me touche,

J'auray parfait en toy l'heur de ma vie,

Et toy en moy l'heur d'estre bien servie

D'esprit, de coeur, d'oeil, de foy et de bouche. ${ }^{5}$

(III/XXXIII)

Il est à remarquer que cette fois-ci l'adorateur s'élève, par la force exaltée de son esprit, jusqu'à l'Idée génératrice de la Beauté, laquelle à son tour sous-tend la beauté de l'aimée. Qu'est-ce à dire, sinon qu'il retrace en sens inverse l'itinéraire décrit par Ficin; donc, que la beauté de l'aimée n'est même plus le prétexte de l'ascension de l'âme, puisqu'au contraire, l'âme atteint d'emblée l'idéal et tire de celui-ci "le pourtrait... de ta beauté". Ainsi, l'adorateur transcende l'objet corporel de son adoration passée et le recrée à la mesure de son désir spirituel.

Du coup il retrouve le sens et le désir de la liberté: rétrospectivement il se voit "enretté", non malgré lui, selon l'habituel schéma pétrarquiste, mais par suite d'un libre don de lui-même. (Ainsi, pour le chrétien, l'obéissance se confond avec la "liberté des enfants de Dieu"). Enfin, dans les tercets, éclate la présence de ce qui avait manqué tout au long des Erreurs amoureuses: la réciprocité. Dans toutes les traditions philosophiques et religieuses que Tyard a étudiées jusqu'ici, et notamment chez Léon l'Hébreu, Dieu a besoin de l'amour de sa créature. L'insensible bien-aimée du Premier livre des Erreurs amoureuses était, par son incommunicabilité, une fausse image du divin. C'est l'expérience finale, celle concevant l'adoration comme une relation réciproque, même si elle ne peut en parler qu'au conditionnel, qui nous renseigne le mieux sur le sens du sacré chez Pontus de Tyard poète amoureux.

Située en dehors des trois recueils des Erreurs, dans le recueil des Vers liriques où Tyard assembla en 1555 une série de poèmes ne traitant pas de son amour, "L'ode du Socratique" dit directement ce qui n'est le plus souvent qu'implicite dans les Erreurs: combien le poète aspire à un Dieu absolu et pur. Cela se constate d'abord dans la strophe où il imagine un Jupiter christianisé, n'exigeant pas de sacrifices sanglants, et où il défend le concept même de la divinité contre ses pitoyables avatars mythologiques:

Car la deité moquée

Des grossiers entendemens,

Lors ne serait point masquée

Sous les humains vestemens. ${ }^{6}$ 
Mais c'est dans l'invocation du "centre" que gît le véritable tribut à la divinité, lui qui est, selon toute doctrine néo-platonicienne, le coeur même de l'essence divine; et c'est donc à lui que va au premier chefle chant le plus religieux que ce poète ait écrit:

\section{O centre, où sied la bonté}

Et non mobile asseurance,

Fais qu'en ta circonference,

La vagabonde beauté

Des saints raiz de la clarté

De ta lumiere feconde

Incorpore sa couleur,

Rendant la Sphere du Monde

En sa parfaite rondeur.?

La beauté est "vagabonde". Par rapport au centre immobile et constant qu'est le bien suprême, c'est-à-dire Dieu, elle est périphérique et mouvante tout en tirant son être de Lui. Les "saints raiz" de la lumière divine sont autant de rayons émanant de Lui et recevant de Lui leur "couleur", c'est-àdire la substance dont les variations produiront leurs qualités sensibles. Dieu, étant cause de tout, l'est aussi de ces qualités sensibles, tout en étant libre d'elles, en tant que centre par opposition à la périphérie. C'est donc l'ensemble infiniment nombreux de tous les rayons partant du centre divin vers la périphérie du divers et de la multiplicité qui forme l'univers. C'est dans ce cadre poétique et philosophique qu'il faut percevoir la situation de l'adorateur humain de Dieu, adorateur tiraillé entre la périphérique "beauté vagabonde" et l'appel absolu du centre.

Donc, que ce soit vis-à-vis de Dieu ou de l'impassible bien-aimée, la poésie tyardienne des années 50 donne du sacré une image à la fois exigeante et sévère. Ce qui n'y est que suggéré se trouve, en revanche, explicité dans le Solitaire premier. Ce dialogue, qui s'annonce au premier abord comme un traité concernant la poésie, n'a en réalité rien d'un "art poétique"; il touche plutôt à la manière dont la poésie sert d'intermédiaire entre l'homme et Dieu: selon la doctrine des quatre fureurs, elle sert à libérer l'âme de ses entraves matérielles et à la réacheminer vers Dieu. Ainsi débute le trajet des vies consacrées à la recherche et à la contemplation des plus hautes valeurs. "Or il est certain que tous ceux, qui ont juré à la louable entreprinse de monter au sommet peu accessible de tant haute montaigne, qu'est la difficile cognoissance de la divinité, cherchans l'un deçà, l'autre delà, qui un endroit, qui un autre plus commode, et aisé, font diverses rencontres de choses, neaumoins rare, et precieuses, comme rien ne se peut trouver autre en lieu tant rare et precieux.... De tous ceux ....qui ont tasché de s'acquerir l'intelligence des choses celestes et divines, et acheminer leurs entendemens jusques au plus hault siege, où repose l'object de 
l'eternelle felicité, les voyes ont esté diverses, commes les doctrines, disciplines, sciences et arts leur ont esté devant les yeux diversement presentez." La contemplation qu'envisage Tyard n'est donc pas une contemplation passivement mystique mais une connaissance active et multiforme. Car si Dieu est un, si le centre est immobile et auto-suffisant, infiniment nombreux sont les aspects de la connaissance qui, tous, mènent à Lui.

Le sacré, à ce stade de la pensée de Pontus de Tyard, n'est donc pas l'absolument autre, en ce sens que Dieu (la connaissance de qui est ici représentée par une "haute montaigne", alors que dans "L'ode du socratique" Il était désigné comme le centre), malgré la distance infinie qui sépare l'entendement humain de Lui, n'est pas inatteignable. Le domaine des "Muses", symboliquement décrit dans le Solitaire premier, représente l'ensemble des médiations (philosophiques, artistiques, scientifiques, sans oublier celles à proprement parler religieuses) au travers desquelles se réalise le désir de s’approcher de Lui.

***

Les Homilies de Tyard incarnent évidemment, de sa part, un profond changement d'orientation comme de genre; elles ne laissent pas, pourtant, de manifester certaines continuités. L'une de celles-ci a trait à l'attitude de l'évêque de Chalon à l'égard de la transcendance. Dans les Homilies, le sens du sacré se nourrit de la différence absolue entre Dieu et l'homme, mais ne va jamais jusqu'à un sentiment de terreur devant l'Éternel.

Dans le cas du premier livre d'homélies, intitulé Homilies ou Discours sur l'Oraison dominicale (Paris: Mamert Patisson, 1585), le genre comme le sujet même invitent à la familiarisation. L'homélie présuppose un climat amical, voire intime entre le prédicateur et son auditoire. La prière dominicale elle-même commence par invoquer Dieu dans son rôle de Père; or, en vertu de la proximité du nom et de la chose dans la vision tyardienne, Dieu est réellement père aux yeux du prédicateur. La paternité de Dieu est liée à sa puissance de créateur, universelle et cosmique, dont proviennent logiquement tous les traits de la paternité au sens charnel. Mais il y a plus: c'est de l'homme que Dieu attend le parachèvement de son amour paternel, et c'est là une preuve, non d'imperfection mais, au contraire, de la plus haute perfection. Ce n'est pas à dire qu'il s'agisse d'une paternité indulgente; Dieu est un père à la fois exigeant et sensible à la disposition intérieure de son enfant, dont la vocation est de lutter à tout moment contre tous les obstacles spirituels et charnels le séparant du père. Le plus important, donc, est ce processus de spiritualisation permettant au fils, même si c'est au travers de difficultés infinies, de s'approcher du père ... Au cours des décennies la réflexion tyardienne a donc maintenu constante l'exigence de spiritualisation requise de l'adorateur; mais elle s'est ouverte 
bien davantage à la dignité humaine, capable de s'aventurer à la rencontre de Dieu, dans la tradition de la Renaissance.

La méditation de la quatrième homélie, qui porte sur le nom de Dieu, contribue également à rapprocher l'humain du sacré. Le pouvoir de nomination est précisément un des terrains où l'homme est co-ouvrier avec Dieu. Dans la Bible, Tyard perçoit deux "nominateurs", Dieu et Adam, imposant à l'origine du monde une double série de noms; la première, qui à l'orée de la création nomme les éléments les plus fondamentaux et la seconde qui, s'insérant dans la marche du temps, désignera le contenu de chacun d'eux. Ainsi, l'homme est admis à partager, sinon l'œuvre de création, du moins celle qui ordonne la création: complémentarité qui ne contredit pas l'absolu de la transcendance symbolisée par le tétragramme. Il est significatif que ce nom suprême, désignant "Dieu par son essence, et non par ses effets", ${ }^{8}$ soit aussi celui qui demeure inconnaissable à la pensée humaine. Mais, de même que les autres attributs de Dieu se déploient au travers de ses autres désignations, Shadai, Elohim, Kadosh etc... de même la pensée de l'homme peut y puiser une connaissance partielle de la divinité. Comme le poète, le théologien traque, au travers des mots, l'indicible. "Que ton nom soit sanctifié" signifie, aux yeux de Tyard, que la réalité de Dieu, mystérieuse et inconnaissable, est par là même suprême et doit être reconnue comme telle.

Prise hors de son contexte, cette méditation sur l'inaccessibilité de Dieu indiquerait que Tyard ne s'est pas départi de son sentiment d'une distance infinie entre l'homme et la divinité. Mais l'ensemble des méditations portant sur l'ensemble de l'oraison dominicale modifie le rôle de l'inaccessibilité divine; en particulier, la spiritualisation requise de l'humain, et l'Incarnation sur laquelle Tyard a tant médité, contribuent, sinon à réduire la distance - dans l'esprit de l'ancienne Alliance elle ést indissolublement liée à l'essence divine - du moins à mettre en valeur le rôle médiateur de Jésus-Christ au sein de la nouvelle Alliance. À la suite des Homilies ou Discours sur l'Oraison dominicale (1585) viennent en effet, coup sur coup, les Homilies, ou Contemplations sur la Passion de Nostre Sauveur Jesus Christ et le Troisième Livre des Homilies . . . sur la dignité de la Croix (1586). C'est donc un moment d'intense concentration sur la souffrance humaine du Christ, solution extrême au problème de la distance extrême. "Ce mesme Christ qui du haut de ceste Croix, ietta l'oeil en bas sur sa mere temporelle, et charnelle, \& sur son cher disciple: haussa de ce mesme lieu sa voix au Ciel, en invoquant Dieu son pere celeste, \& eternel [ ....] Ce mesme Christ, et verbe divin, qui avoit creé le Soleil et la terre, a donné en la Croix, cause supernaturelle au Soleil, de s'obscurcir: \& en la terre, de trembler universellement. Bref ce mesme Christ, qui est au Ciel, de naissance eternelle, a souffert mort temporelle en la Croix. Voyez donc si ceste Croix ne doit pas estre tenue pour riche conque, \& nacre recherchable: puis qu'elle a porté la 
precieuse et inestimable perle, $\&$ vraye union divine Iesus Christ nostre Sauveur, en toutes les conditions qui peuvent estre attribuees en la vraye et singuliere union de son humanité, à sa divinité."9

Nous y voilà; c'est par la souffrance du Christ que, dans la vision de Pontus de Tyard, la distance est abolie, mais non le sacré. Nous assistons dans ces homélies au processus de sacralisation de quelque chose qui fut infiniment familier au poète: le martyre d'un homme. Mais il ne s'agit plus du martyre métaphorique de l'amant pétrarquiste que fut le poète contemplant une déesse inaccessible. C'est maintenant un Dieu souffrant qui par son sacrifice invite le regard et appelle l'amour de celui qui le contemple. Renversement des rôles, dont parle ici même Robert Melançon à propos de La Ceppède, et dans lequel la souffrance elle-même devient sainte. C'est ainsi que l'homélie répond, chez Pontus de Tyard, à sa poésie d'antan.

\section{Université McGill}

\section{Notes}

1 Pontus de Tyard, poète et philosophe (en préparation).

2 Maurice Scève, Delie, obect de plus haulte vertu, édition critique par Eugène Parturier (Paris: Hachette, 1916), p. 58.

3 Ibid., p. 115.

4 Pontus de Tyard, Euvres poétiques complètes, éd. John C. Lapp (Paris: Didier, 1966), p. 12.

5 Ibid., p. 153.

6 Ibid., p. 174.

7 Ibid.

8 Pontus de Tyard, Homilies ou Discours sur l'Oraison dominicale, dans Trois livres d'Homilies (Paris: Mamert Patisson, 1586), p. 41.

9 Pontus de Tyard, Trois livres d'Homilies, p. 252. 